\title{
A malpractice: Stripping of the great saphenous vein without the division of the side-branches at the saphenofemoral junction
}

\author{
Alat I \\ Department of Cardiovascular Surgery, Afyonkarahisar State Hospital, Afyonkarahisar, Turkey. \\ ilkeralat@hotmail.com
}

\begin{abstract}
AIM: To highlight the components of stripping operation of the great saphenous vein and to offer a proposal for guidelines.

METHODS: 7789 admissions with venous insufficiency during the period, reaching seven and a half years were evaluated. Seventy- two admissions of nineteen patients were related to the recurrent symptoms due to previous incomplete stripping surgery. Doppler ultrasonography evaluations were made. The remained venous segment from the first operation was excised in the second operation.

RESULTS: Mean duration between two operations was 7.44 years. Preoperative clinics were changing between $\mathrm{C} 2 \mathrm{~s}$ and $\mathrm{C} 5$ according to CEAP classification. Only nine patients could be persuaded to undergo the second operation. The remaining six patients rejected the second operation.

CONCLUSION: While the patients who were operated on for the second time regained their health, others became the epitome of hopelessness and mistrust. They lost their confidence in medicine and surgery. If a stripping operation is planned, it should be performed in full accordance with the surgical procedure of stripping as mentioned in the classical textbooks. Guidelines should contain expressions reminding of ethical issues. This will prevent the dereliction of the duty and the loss of money, labor, time, health, patients' confidence in surgery (Tab. 1, Fig. 1, Ref. 18). Text in PDF www.elis.sk.

KEY WORDS: ethics, malpractice, quality of life, venous, saphenous, stripping.
\end{abstract}

\section{Introduction}

In the process of medical practice carried out by people other than those engaged in the ethics of science, even if the ethical issues are ignored and evaluated by those practitioners as if they are stable and fixed, in parallel with the developing technology, the ethical rules should be checked and renewed frequently due to the changing medical practices. For these reasons, it is imperative that those people who are the real and professional owners of the science of ethics have to be involved in the preparation and follow-up of ethical issues in daily medical practice. It is unfortunate and unsuccessful for the ethics committees, who are selected from people who are doing their own daily medical practices, to follow the ethical aspects of these jobs or to take part in the preparation of ethical rules.

In this retrospective study, a case of malpractice, which is increasingly seen recently regarding the stripping surgery involved in the treatment of great saphenous vein (GSV) insufficiency, will be discussed. And the negative situations caused by this malpractice application on patients and the ethical practices that may have a role in the elimination of these conditions will be discussed.

Department of Cardiovascular Surgery, Afyonkarahisar State Hospital, Afyonkarahisar, Turkey

Address for correspondence: I. Alat, Dr, Afyonkarahisar Devlet Hastanesi, Kalp ve Damar Cerrahisi Klinigi, Afyonkarahisar, Turkey.
Flush ligation and stripping of the GSV, as advocated by Myers nearly 60 years ago (1), has been the accepted as standard of management for symptomatic varicose veins (2). Although the clinical outcomes of some reports suggest that endovenous laser ablation (EVLA) (3), radiofrequency ablation (4), should be considered as the treatment of choice for suitable patients, a different group of manuscripts reported that a significantly higher varicose vein recurrence rate was observed at the saphenofemoral junction (SFJ) region after EVLA compared with stripping $(5,6)$. It has been estimated that the statistical difference between the outcomes of stripping and EVLA must be related to operative difference at the SFJ region. As described by Myers, in a classical stripping operation, the GSV must be flush ligated with the femoral vein (FV) at the junction and all other veins coming into the FV laterally and medially should be ligated and removed. Likewise, the medial and lateral superficial branches of the GSV should be ligated and removed (1).

However, in our daily practice, it is possible to encounter some patients with recurrent varicose veins after stripping. Ultrasonographic evaluations of these patients have proved that flush ligation and stripping of the GSV were not performed properly, although they have patient reports, indicating their previous stripping operation.

In this retrospective report, the outcomes of the patients who underwent incomplete stripping operation previously are presented together with the results of their second operation due to recur- 
rent symptoms of venous insufficiency. In addition, reasons for previous incomplete surgery are discussed from the perspective of ethics and proposals are offered for guidelines.

\section{Materials and methods}

\section{Patients}

Patients in this retrospective report are the consecutive patients of the author of this manuscript. An ethical approval was obtained from the institutional board and authorities. Informed consent was obtained from each patient.

7789 admissions with the symptoms of venous insufficiency were listed in the hospital records of the author's outpatient clinic during the period, reaching seven and a half years. Seventy two admissions of this amount were related to the recurrent venous insufficiency symptoms due to previous incomplete stripping surgery, performed by different surgeons in the different centers. These 72 admissions belonged to 19 patients. Moreover, the number of 72 was only the number of the applications of the patients to the outpatient clinic of the author of this manuscript. However, when the number of applications of these patients, made to different cardiovascular surgeons in other hospitals over the years due to dissatisfaction after their initial operations is taken into consideration, total applications number is over several hundreds. The findings of 15 patients whose records were reached were included in the study.

\section{Preoperative evaluation}

In addition to their physical examinations, all of the patients were evaluated with the colored Doppler ultrasonography (CDU). All parts of their venous systems, particularly the SFJ regions were evaluated with the CDU. Epicrises were obtained from the patients who underwent stripping operations previously.

\section{Surgical operation}

After giving the information about the second operation, informed consents were obtained from all of the patients. After suitable local anesthesia with prilocaine, the SFJ region was explored through a three-cm-incision at the groin. The remained GSV segment from the first operation was excised after all side branches were ligated and removed. Finally, a flush ligation of the GSV was obtained.

\section{Medications and follow-up}

Patients underwent antibiotic prophylaxis starting one hour before surgery and completing by the end of the first week after surgery. Furthermore, $150 \mathrm{mg}$ of acetylsalicylic acid per day and classical venotonic agents were ordered postoperatively. A compression sock was suggested postoperatively. The operated extremities of the patients were elevated during the first night after surgery. All patients were discharged one day after their operation. To determine the changes in their quality of life, they were asked about their feelings and the existence or absence of their complaints before discharging.

\section{Statistical analyses}

For statistical analyses, "Paired t test" was used in order to determine the patient satisfaction and the effect of second operation on the patients' feeling and symptoms. Fisher Exact Test was used to determine if there was any difference between the confidence of the patients in the surgery who were operated and those who were not. Any p value less than 0.05 was assumed statistically significant.

\section{Results}

\section{Preoperative examinations}

Nineteen patients with the recurrent symptoms of venous insufficiency after previous incomplete stripping operation were admitted to author's outpatient clinic during the period, reaching seven and half years. The total number of applications made by these patients to the author's outpatient clinic was 72 . However, when the number of applications of these patients, made to different cardiovascular surgeons in other hospitals over the years due to dissatisfaction after their initial operations is taken into consideration, total application number is over several hundreds. It is not precisely possible to find and calculate retrospectively the unnecessary hospital expenses and time losses associated with these hospital re-admissions. Moreover, these financial losses are not only the financial losses of the patients but also the financial losses of the insurance companies.

The findings of 15 patients whose records were reached were included in the study.

Only nine of these fifteen patients could be persuaded to undergo the second operation. Neither their painful clinics nor medical explanations could induce the rest of the patients to undergo the second operation. These six patients never agreed to undergo any re-operation. However, these six patients who rejected second operation can not be excluded from this report. These six patients are important as much as other nine patients who underwent secondary operation. The nine operated patients are the evidence of showing the importance of ligation and division of the side branches at the SFJ region. Because all of the complaints of these patients were completely resolved after their second operation. On the other hand, six patients who rejected secondary operation are the evidence of hopelessness and mistrust. They no longer rely on medicine or surgeons. This is an important outcome of previous incomplete stripping operation. This is also the proof of how sorrowful is the abandonment of the ethical rules when performing an operation. Patients of this article teach us that if any surgical procedure is performed outside the code of ethics, it will result in two different outcomes: Firstly, they will have to struggle with

Tab. 1. Patients' statistical information.

\begin{tabular}{lccccc}
\hline & Min & Max & Mean & Median & SD \\
\hline Patients' ages (years) & 31 & 65 & 42.11 & 53 & 11.59 \\
\hline $\begin{array}{l}\text { Time elapsed between } \\
\text { two operations (years) }\end{array}$ & 3 & 14 & 7.44 & 7 & 3.13 \\
\hline $\begin{array}{l}\text { Follow up time after the } \\
\text { second operation (months) }\end{array}$ & 8 & 61 & 43 & 52 & 17.1 \\
\hline
\end{tabular}




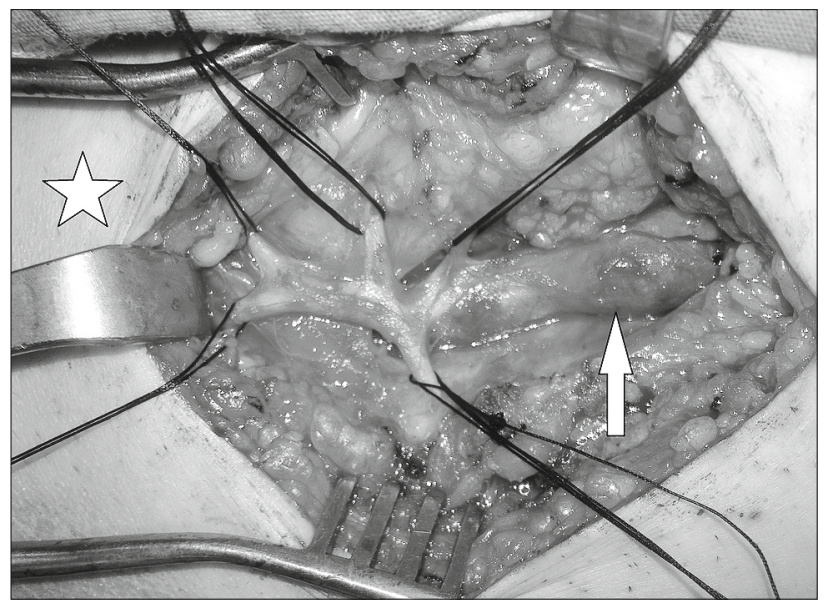

Fig. 1. A scene from the second operation before ligations. As seen in the photograph, none of the side branches of the SFJ region were divided or ligated in the previous stripping operation. Additionally, the GSV already exists in its native location. The stump of the GSV is longer than $2 \mathrm{~cm}$ as seen. The white arrow indicates the GSV. Silk sutures were passed around all side branches for ligation. The figure of star indicates the head side of the patient.

the remaining part of organic disorders related to their previous disease and secondly their mental and spiritual structure will be affected negatively.

It was observed that there is a statistically significant difference between the operated and un-operated patients with regard to their confidence in surgery and medicine $(\mathrm{p}<0.001)$. The patients who refused the second operation lost their confidence in surgery and medicine.

\section{Demographic data}

One of the operated patients was female and the rest of them were male.

The statistical values of the patients' ages and the statistical data related to the time elapsed between two operations are summarized in Table 1.

Patients' preoperative clinics were changing between C2s and C5 according to the CEAP classification (C2s-C5, Ep, As \pm d, Pr).

According to their CDU examination, all patients had a Grade-4 reflux (A reflux longer than $4000 \mathrm{~ms}$ ) at their SFJ regions.

\section{Operative findings}

In all patients, it was observed that the GSV was already existing in its native location and all side-branches were untouched. An operative view of a patient is presented as an example in Figure 1.

\section{Postoperative findings}

Statistical analyses of follow-up time for the second time operated patients are presented in Table 1.

In the postoperative period, symptoms of all patients resolved. Their clinical signs decreased to C2a according to CEAP classification. All patients expressed their satisfaction. When comparing the preoperative feelings and thinking of the operated patients to their postoperative conditions, the patients stated they were feeling much better postoperatively ( $\mathrm{p}<0.001)$.

Especially, the medical history of one of the patients was extremely remarkable. Before his second operation, he had to be hospitalized frequently and was forced to get a two-month-rest report per year for years. His preoperative score was 17 according to Venous Clinical Severity Score (VCSS). Preoperatively, he had a severe cellulitis with an effluxion from his skin, despite of combined antibiotics. After his second operation, the cellulitis on his leg decreased remarkably, he started to work regularly. His VCSS was 7 points postoperatively. In other words, as a result of his previous incomplete surgery which was performed outside the code of ethics, this patient had to deal with microbial diseases for years, attempted to be healed with many antibiotic therapies, had been exposed to the side-effects of these drugs. Additionally, he had lost significant amount of his money and wasted his time and had lost workforce due to mandatory rest-reports. But he was still lucky because he accepted the second surgery and his life turned back to normal again. However, the patients in the second group that refused the second surgery were still dealing with the problems of their diseases and most importantly they had no confidence in medicine and surgery. Moreover, it was not only with regard to the second operation for their present disease, but also it seemed that it was really hard to convince them of any treatment in any area of medicine or surgery.

\section{Discussion}

Nearly 60 years passed since Myers published his report about stripping operations. He stated in his report that the GSV must be flush ligated with the FV at the junction and all the other veins coming into the FV laterally and medially should be ligated and removed. Likewise, the medial and lateral superficial branches of the GSV should be ligated and removed (1). Years after Myers, although conventional stripping operations were mentioned in the classical textbooks together with the ligation and division of all of the side branches of the GSV (2), seeing incomplete stripping operations without medical reasons is really disappointing.

According to the Clinical Practice Guidelines of the European Society for Vascular Surgery (ESVS), the Recommendation 48 (Surgical stripping of the saphenous vein without high ligation leaving a $2 \mathrm{~cm}$ stump may be considered.) corresponds to Class IIb and Level B. (Class IIb: Usefulness/efficacy is less well established by evidence/opinion. Level of evidence B: Data derived from a single randomized clinical trial or large non-randomized study) (7).

These determinations express that any stripping operation without high ligation does not have a world-wide acceptance. In that case, why does a surgeon perform a stripping operation without flush ligation of the GSV and why does he/she leave the side-branches untouched although he/she is working near them? Might this have a medical reason? Or could it have an explanation within the boundaries of ethical science?

Unfortunately, answering these questions scientifically is not always possible , because everyone is not always honest and will not speak honestly. However, it's the observation of the 
author of this manuscript that some surgeons - for only shortening the duration of the operation- have developed a habit such as quitting the division of the side-branches at the SFJ region. Whereas, dereliction of a duty can not be accepted. This is an ethical problem. Likewise, it was observed that some surgeons are neglecting the division of the side-branches for managing to operate much more patient in the same operating theatre, if it is a common-used operating theatre particularly. None of these applications is acceptable ethically. Because negligence is -ethicallyone of the biggest crimes and it finds a place in the definition of "malpractice".

At this point, the report of Woodyard (8), has really an important evaluation. In the Woodyard's report, the author categorized events that could result in negligence of a surgeon as follows: 1- Neglecting the basic principles of surgery, 2- Being too busy, 3- Inexperience, 4- Failure of communication. When we look at the classification in this report, we see that the case of 'the neglect of a surgical principle' is considered as the first of the reasons for the negligence of the surgeon. That confirms the thesis of this manuscript that incomplete stripping surgeries should be considered as a negligence and therefore a malpractice because the fact that this surgery is performed nowadays outside of the principles first described previously. Moreover, the concept of negligence mentioned under the title 'being too busy', which is considered in the second order in Woodyard's classification, fully coincides with the fact that some of the surgeons, as we have described in our study, doing incomplete surgeries in order to get more patients in the same hall. As a result, because of neglecting some part of surgery in contrary to what was first described, it is not unreasonable to define incomplete stripping surgeries as a 'malpractice'. Furthermore, it's an ethically justified discourse.

However, the main issue should be to prevent scientifically the possibility of the occurrence of these unethical events. For this purpose, special comments and statements as follows should be included in the venous guidelines: "It's recommended that flush stripping of the GSV with the division of the side-branches at the SFJ region should be considered together unless there is an absolute medical contraindication for the division of the side-branches. Otherwise, stripping of the GSV without the division of the sidebranches will be considered as a malpractice.”

According to the literature, incomplete removal of the GSV is the most common cause for recurrent varicose veins (9). Cina and co-workers indicated that the main cause of varicose recurrence is inadequate surgery (10).

Additionally, MacKenzie and co-workers reported that failure to strip is frequently associated with the development of new deep venous reflux and the benefits of routine stripping operation may relate to a favorable impact on deep venous function (11).

In another manuscript, Eris and coworkers reported a total of 287 legs of 247 consecutive patients who were re-operated due to recurrent varicose veins and residual saphenous vein was recorded in 183 legs (64\%) (12).

As seen in all the manuscripts above, residual saphenous veins play a big role in the etiology of recurrent varicose veins. Therefore, it is not only an ethical problem to allow incomplete stripping surgeries or to keep silent, but also leads to organic-anatomical damages that have a negative impact on the patient's health.

On the other hand, if the articles, comparing EVLA with stripping procedure are examined, it's possible to see that stripping operations have a superior effect to EVLA as long as high ligation of the GSV (HLS) is added to stripping. In terms of same site clinical recurrence and saphenofemoral refluxes, HLS is superior to $\operatorname{EVLA}(5,6,13-15)$. So, this is another evidence indicating that flush ligation of the GSV is the most important factor for the success of stripping operations.

By the way, even if it seems that the results of incomplete stripping are same with the endovenous ablation procedures (EVAPs) including EVLA, there is a quite distinction between them in regard to the ethical aspect. To do an incomplete surgery without any scientific reason is a malpractice and must not be forgiven. Incomplete stripping operation is such a thing. However, EVAPs are not a product of malpractice. Yes, both have similar results in the patients' venous hemodynamic parameters, but the situation in EVAPs is related to the technical limitations of the procedures only. There is not an additional chance to do much more or to ablate saphenofemoral junction for a surgeon, planning to do any type of EVAPs. So, during the period of the surgery planning, the surgeon and his/her patients know and accept that something will be lack or missing. Anyway, for a particular group of patients, EVAPs can be a solution and sometimes only a single one solution.

However, to neglect the division and ligation of the sidebranches at the saphenofemoral junction during a stripping operation without any scientific reason can not be explained with the limitation of the procedure. So, this is obviously a malpractice.

On the other hand, according to the previous literature $(5,6$, 13-15), even if stripping operation has superior effects than EVAPs because of the ligations of the side-branches at saphenofemoral junction, anyway, as being the scientists and/or surgeons, we must not abandon EVAPs. We shouldn't close our eyes to the developments, innovation and science. But there is also an essential point here that we shouldn't hesitate to report the missing and inadequate situations due to EVAPs either.

Before EVAPs is fully mature, no one should try to popularize EVAPs more than it deserves. This is another ethical side of the issue. Actually, this is not a pessimism. In contrast, to accept and to report the limitations of the EVAPs and negative reports indicating inadequate or recurrent surgeries due to EVAPS are all crucial to further developing EVAPs' technologies. For example, we were not able to do venous thrombectomies up to the recent past because of the pulmonary embolism after the procedure. Then, we, mankind, invented caval filters and combined their application with the percutaneous venous thrombectomy procedures and now we're able to do venous thrombectomies with a reasonable mortality rates. Was there anyone who was dreaming of percutaneous venous thrombectomies centuries ago?

If we don't hesitate to announce negative reports due to EVAPs, it's possible that we can force someone to discover some new materials and EVAPs might be fully matured.

In conclusion, EVAPs can not be defined as a malpractice in contrast to incomplete stripping operations. We should try to have 
242-247

EVAPs' technologies matured. For this purpose, we shouldn't try to show them better than they are and we shouldn't hesitate to report their limitations and shouldn't try to popularize them much more in the guidelines than they deserve.

At this point, the importance of the science of ethics becomes clearer. As it is well known, the history of medical ethics has major turning points: centuries to century $(16,17)$. In the past, it was possible that these major turning points of ethics could be clearly defined. However, due to the rapidly developing and changing technologies nowadays, medical and surgical therapies have been changing in shorter periods. Therefore, it is not easy to define these turning points for ethical science at present. Thus, professionals on the science of ethics should work collaboratively with vascular surgeons, they should follow these developments in the area of vascular surgery and they should follow the patients in their preoperative and postoperative periods and determine the ethical margins of these applications. And all these works should be followed not by the ethical committees formed by those who carry out these operations, but by the boards formed by professional ethics scientists themselves.

In the light of the results of the above articles it is certain that a stripping operation has a greater value if it is made together with the ligation and division of the side branches. Otherwise, recurrent symptoms are frequent and this incomplete work will also destroy the other parts of the lower venous systems in the future. Finally, neglecting some part of the duty will result in a waste of time, labor, money and health.

The outcomes of this manuscript is the evidence for the statements above. The results of the operated nine patients are extremely remarkable. Their clinical status recovered according to the CEAP classifications. Particularly, the case mentioned in details in the Results section showed significant improvement. Despite of nearly five years after his second operation, he has been still working regularly, whereas he was not able to go to work due to the complications of previous incomplete stripping operation before the second surgery. Previously he had to get rest reports frequently. Similar results were obtained from other operated patients.

On the other side, patients who rejected secondary operation are the evidence of hopelessness and mistrust. They no longer rely on medicine or surgeons. Neither their painful clinics nor medical explanations could induce them. Although dozens of times in years they had applied to many different surgeons in different hospitals, it was not possible to convince these patients of treatment or of a new operation. This is another important outcome of previous incomplete stripping operation. Who and how will reassure their lost confidence in medicine and surgery? When these patients have a life-threatening disease, will they accept any medical or surgical intervention? This subject should be a topic for further investigations. The spiritual and reflective side effects of incomplete stripping operations on the patients' habits should be investigated and their behavior pattern should be observed particularly when they are sick.

Moreover, all these problems are related to only the patient's side of the event. However, there is also another aspect of the event facing the patient's relatives. When the literature is exam- ined from this perspective, it's possible to see the reports of the diseases about not only the negative effects on patients, but also the reports stating the effects of the diseases on the quality of life of their first-degree relatives (18). Unfortunately, this aspect of the disease related to the family of the patient is not often questioned. It would be correct to evaluate the problems related to patient relatives in two separate plans: A-Inside the house and B-Outside the house. When our patient who had to get frequent rest-reports is taken into consideration, it is inevitable that he had experienced serious problems at home due to his frequent rest-reports since he was responsible for providing the needs for his family. This is an example for the ones inside the house (A). On the other hand, all patients who rejected the second operations and accepted living with their health problems are such as they are like a walking billboard declaring negative advertisements. These patients who were left alone with some problems due to incomplete surgery, not only lose their confidence in medicine and surgery, but also impose these negative thoughts on their immediate environment. Therefore, insecurity to medicine and surgery has been gradually growing as an epidemic disease due to this patient group. This is an example for the ones outside the house (B). Therefore, the negative impacts of these patients who have incomplete stripping surgery on the quality of life of their own households and their immediate surroundings should be considered as a separate study. As a result, it can be said that incomplete stripping surgery has negative effects not only on the patient himself/herself but also in many areas on his/her household, immediate environment and insurance companies.

\section{Conclusion}

If a stripping operation is planned for a patient with venous insufficiency, this surgery should be performed in full accordance with the surgical procedure of stripping as mentioned in the classical textbooks unless there is an absolute contraindication to do it.

Guidelines should contain expressions reminding ethical issues. A statement such as following can be included in the guidelines: "It's recommended that flush stripping of the GSV with the division of the side-branches at the SFJ region should be considered unless there is an absolute medical contraindication for the division of the side-branches. Otherwise, stripping of the GSV without the division of the side-branches will be considered as a malpractice.”

Any expression like above in the guidelines will prevent the dereliction of the duty. So, this will result in greater satisfaction of the patients and also prevent the loss of money, labor, time, health and patients' confidence in medicine and surgery.

However, these arrangements do not seem to be possible with unilateral organizations. Collaborative work between professionals on ethics and surgeries have a great importance and there is a serious need for that.

\section{References}

1. Myers TT. Results and technique of stripping operation for varicose veins. JAMA 1957; 163 (2): 87-92. 
2. O’Donnell TF \& Iafrati MD. Varicose veins. In: Haimovici, H, Ascer E, Hollier LH, Strandness DE, Towne JB eds. Haimovici's Vascular Surgery Principles and Techniques. Fourth Edition. Massachusetts: Blackwell Science, Inc., 1996; 1187-1198.

3. Nandhra S, El-sheikha J, Carradice D, Wallace T, Souroullas $P$, Samuel N, et al. A randomized clinical trial of endovenous laser ablation versus conventional surgery for small saphenous varicose veins. J Vasc Surg 2015; 61 (3): 741-746.

4. Morrison N, Gibson K, McEnroe S, Goldman M, King T, Weiss R, et al. Randomized trial comparing cyanoacrylate embolization and radiofrequency ablation for incompetent great saphenous veins (VeClose). J Vasc Surg 2015; 61 (4): 985-994.

5. Gauw SA, Lawson JA, van Vlijmen-van Keulen CJ, Pronk P, Gaastra MT, Mooij MC. Five-year follow-up of a randomized, controlled trial comparing saphenofemoral ligation and stripping of the great saphenous vein with endovenous laser ablation (980 nm) using local tumescent anesthesia. J Vasc Surg 2016; 63 (2): 420-428.

6. Rass K, Frings N, Glowacki P, Gräber S, Tilgen W, Vogt T. Same site recurrence is more frequent after endovenous laser ablation compared with high ligation and stripping of the great saphenous vein: 5 year Results of a Randomized Clinical Trial (RELACS Study). Eur J Vasc Endovasc Surg 2015; 50 (5): 648-656.

7. Wittens C, Davies AH, Bækgaard N, Broholm R, Cavezzi A, Chastanet S, et al. European Society for Vascular Surgery. Editor's Choice Management of Chronic Venous Disease: Clinical Practice Guidelines of the European Society for Vascular Surgery (ESVS). Eur J Vasc Endovasc Surg 2015; 49 (6): 678-737.

8. Woodyard JE. Negligence and the surgeon. Ann R Coll Surg Engl 1980; 62 (6): 476-478.

9. Joshi D, Sinclair A, Tsui J, Sarin S. Incomplete removal of great saphenous vein is the most common cause for recurrent varicose veins. Angiology 2011; 62 (2): 198-201.
10. Cina G, Narese D, Amicucci V, Filipponi M, Snider F. Recurrent varicose veins of the legs: a single centre experience. Clin Ther 2014; 165 (4): 183-186.

11. MacKenzie RK, Allan PL, Ruckley CV, Bradbury AW. The effect of long saphenous vein stripping on deep venous reflux. Eur J Vasc Endovasc Surg 2004; 28 (1): 104-107.

12. Eris C, Yavuz S, Gucu A, Yumun G, Toktas F. Recurrent varicose veins after surgery: an analysis of 247 patients. Turk Gogus Kalp Dama 2014; 22 (2): 347-353.

13. Kalteis M, Adelsgruber P, Messie-Werndl S, Gangl O, Berger I. Five-year results of a randomized controlled trial comparing high ligation combined with endovenous laser ablation and stripping of the great saphenous vein. Dermatol Surg 2015; 41 (5): 579-586.

14. Smith PC. Debate: should persistent incompetent truncal veins be treated immediately? The case in support of the statement. Phlebology 2015; 30 (1 Suppl): 107-110.

15. Flessenkämper I, Hartmann M, Hartmann K, Stenger D, Roll S. Endovenous laser ablation with and without high ligation compared to high ligation and stripping for treatment of great saphenous varicose veins: Results of a multicentre randomised controlled trial with up to 6 years follow-up. Phlebology 2016; 31 (1): 23-33.

16. Namm JP, Siegler M, Brander C, Kim TY, Lowe C, Angelos P. History and evolution of surgical ethics: John Gregory to the twenty-first century. World J Surg 2014; 38 (7): 1568-1573. doi: 10.1007/s00268014-2584-1.

17. McCullough LB. Laying medicine open: understanding major turning points in the history of medical ethics. Kennedy Inst Ethics J 1999; 9 (1): 7-23.

18. Macho P, Bohac M, Fedeles J Jr, Fekiacova D, Fedeles J Sr. Impact of cleft lip and/or palate in children on family quality of life before and after reconstructive surgery. Bratisl Med J 2017; 118 (6): 370-373.

Received October 8, 2019. Accepted November 29, 2019. 